Palabra Clave (La Plata), octubre 2017, vol. 7, n 1, e042. ISSN 1853-9912

Universidad Nacional de La Plata.

Facultad de Humanidades y Ciencias de la Educación.

Departamento de Bibliotecología

\title{
Las Conferencias: espacio y tiempo de aprendizajes en la Biblioteca Médica Nacional de Cuba
}

\author{
Conferences: space and time for learning in the \\ National Medical Library of Cuba
}

\section{Maria del Carmen González Rivero *}

* Biblioteca Médica Nacional de Cuba. Centro Nacional Información Ciencias

Médicas/INFOMED, Cuba I maria.carmen@infomed.sld.cu

\section{PALABRAS CLAVE}

Conferencias

Aprendizaje

Capacitación

Bibliotecarios

Bibliotecas

Alfabetización informacional

Sociedad de la información

\section{KEYWORDS}

Conferences

Learning

Training

Librarians

Libraries

Informacional literacy

Information society

\section{RESUMEN}

Se pretende detallar cómo las conferencias pueden clasificar en los nuevos espacios de aprendizaje a partir de identificar las características que distinguen los tradicionales espacios de enseñanza. Existe una amplia variedad de métodos o técnicas para capacitar al personal que trabaja en bibliotecas. En este caso el interés está dado por mostrar los propósitos y efectividad de las conferencias que se realizan en la Biblioteca Médica Nacional (BMN) todos los martes desde el 2013, con el objetivo de utilizar métodos prácticos y fáciles de ejecutar, siendo una manera rápida y sencilla de proporcionar conocimientos a grupos de bibliotecarios o usuarios, facilitando el aprendizaje a través del uso de recursos audiovisuales, imágenes, gráficos, fotografías, grabaciones de videos o películas. Se muestran conclusiones a partir de estadísticas graficadas que permiten realizar valoraciones.

\begin{abstract}
It tries to be identified like the Conferences they can classify in the new learning spaces from identifying the characteristics that distinguish the traditional education spaces. There exists a wide variety of methods or skills to qualify to the personnel that works in libraries, in this case the interest is given for showing the intentions and effectiveness of the conferences that are realized in the National Medical library (BMN) every Tuesday from 2013, with the target to use methods practical and easy to execute, in a rapid and simple way to provide knowledge to groups of librarians or users, across the use of audio-visual resources, images, graphs, photos, recordings of videos or movies to facilitate learning. Conclusions show themselves from statistics charted that allow to realize evaluations.
\end{abstract}

Recibido: 17 de enero de 2017 | Aceptado: 12 de junio de 2017 | Publicado: 9 de octubre de 2017 


\section{Introducción}

Desde que el hombre primitivo comenzó por vez primera a demostrar a sus semejantes o descendientes la forma de partir una fruta o una rama con el uso de determinado instrumento, estaba haciendo uso de lo que hoy se conoce como tecnología educativa. A pesar de ser un concepto relativamente reciente, la educación ha estado vinculada desde siempre al uso de determinados métodos para el logro de sus objetivos.

Es importante poder definir adecuadamente las diferencias entre métodos y técnicas en la enseñanza, pues si bien ambos están encaminados a un mismo fin, tienen diferentes objetivos y aplicaciones de acuerdo a las características concretas del proceso de enseñanza. Mientras el método es únicamente la forma en que se realiza algo, con orden y siguiendo ciertos principios, la técnica hace referencia a las herramientas que se utilizan para hacer llegar el conocimiento (Spring, Kunkel, Gilman, Henderson y White, 2016).

Entre otras están las técnicas didácticas, que pueden ser técnicas instrucciones (centradas en el instructor: expositiva o conferencia, dialogo-discusión o interrogativa, demostrativa, etc.) y técnicas grupales (centradas en los participantes: lluvias de ideas, mesa redonda, análisis de casos, juego de roles).

Existe una amplia variedad de técnicas para capacitar al personal que trabaja en una biblioteca. Es conocido que ninguna técnica es siempre la mejor; el mejor método dependerá de la efectividad respecto al costo, el contenido deseado del programa, la idoneidad de las instalaciones con que se cuenta, las preferencias y la capacidad de las personas, las preferencias y la capacidad del capacitador, más los principios de aprendizaje a emplear.

Para lograr cumplir objetivos concretos en espacios de aprendizaje se utilizan técnicas que resultan comunes para la capacitación. Se pueden nombrar algunas, como la capacitación en el puesto, que resulta ser un método en el que la persona aprende una tarea o una destreza mediante su desempeño real, y consiste en asignar nuevos trabajadores a los trabajadores experimentados que se encargan de la capacitación real. Esta modalidad capacitadora se complementa con la rotación del puesto, en la que el trabajador pasa de un puesto a otro en periodos programados para conocerlas diferentes actividades que se desarrollan en el proceso general, ya sea en áreas de procesos técnicos o servicios al público. Este método es utilizado para el diseño de los entrenamientos en la Biblioteca Médica Nacional (BMN) cuando llegan los egresados de escuelas técnicas de bibliotecología y son insertados en prácticas laborales en el tiempo comprendido de 1 a 3 años.

Para Petrinic y Urquhart (2007) la capacitación laboral para los egresados de bibliotecología y la orientación o el apoyo en el primer papel profesional en el sector de la salud parece altamente recomendable, como una forma de ayudar a los nuevos participantes a identificar cómo sus habilidades y conocimientos existentes pueden transferirse, y cómo se pueden abordar las deficiencias de conocimientos. Más allá de esa etapa inicial, los profesionales de la información que trabajan en el sector de la salud necesitan capitalizar las oportunidades de adquirir habilidades especializadas y conocimientos de especialistas, ya que les dará más confianza en los nuevos papeles emergentes. 
Otro procedimiento empleado en la citada institución, para capacitar a trabajadores y egresados en tiempo de práctica laboral, son las conferencias, que constituyen métodos prácticos y fáciles de ejecutar y son una manera rápida y sencilla de proporcionar conocimientos a grupos grandes de personas. La conferencia se puede acompañar de materiales impresos para facilitar el aprendizaje; asimismo, se pueden utilizar recursos promocionales a través de imágenes, gráficos, fotografías, grabaciones de videos 0 películas que respaldan y logran hacer más efectivo el mensaje. Los audiovisuales también pueden ser incorporados a las conferencias para reafirmar la idea central que se ha llevado al auditorio.

Siguiendo la ruta de interés que los especialistas a cargo de las áreas de Procesos Técnicos y Servicios al Público de la BMN se han trazado en años, como garantía de elevar la profesionalidad de sus líderes, directivos y técnicos, debe retomarse una actividad que se realizaba de aprendizaje programado. Esta actividad consistía en la aplicación de un método sistemático para enseñar habilidades para un puesto determinado, luego se presentaba un conjunto de preguntas o hechos para que el aspirante respondiera, se revisaba y comparaba con las respuestas y retomaba aquellas en las que se había equivocado, hasta responder correctamente todas.

Este método es efectivo porque permite al trabajador una retroalimentación inmediata sobre la precisión de sus respuestas y sobre el aprendizaje que va logrando.

A este ritmo y ante los nuevos retos que presenta la Sociedad de la Información, las bibliotecas requieren de recursos humanos de alta calidad, que permitan satisfacer las demandas de sus usuarios e impulsar diversas iniciativas para la mejora continua de los servicios y procesos. El personal bibliotecario es un factor esencial; por ello es importante realizar actividades de autopreparacion y mostrar mediante técnicas expositivas los conocimientos teóricos y prácticos, creando de esta forma espacios que permitan compartir con otros colegas las tendencias en determinadas áreas de una biblioteca y su equipo, vinculados a cambios generados por las nuevas tecnologías de la información en el campo de las bibliotecas, así como las nuevas funciones adoptadas por sus especialistas para adaptarse a las exigencias de la Sociedad de la Información.

Ante la interrogante y la necesidad presentada en la BMN para lograr la superación profesional de sus especialistas y técnicos, fue diseñado un espacio capacitador de Conferencias, con el objetivo de comunicar, debatir y llevar a la acción, o ser solamente un espacio de corte informativo, basado en temáticas de actualidad nacional e internacional en el ámbito bibliotecario y las bibliotecas.

Como afirma Hernández Pacheco y Quezada Escamilla (2016), el desarrollo integral de los recursos humanos, va más allá de la adscripción, la designación, el entrenamiento o capacitación y la remuneración; por ello, es vital dotar de una formación continua al personal, en el marco de otras funciones básicas de los recursos humanos como son la planeación estratégica de los puestos, el reclutamiento y la selección basados en aspectos como la escolaridad o la preparación académica, tomando en consideración además las habilidades o competencias para el trabajo. Asimismo, los autores señalan es importante tener en cuenta la evaluación del rendimiento y del potencial, la promoción del personal o desarrollo de carreras, la comunicación efectiva y el clima laboral, la administración del 
talento, la ergonomía y la prevención de riesgos laborales, así como las relaciones humanas y laborales, entre otros aspectos.

Los martes de Conferencia en la Biblioteca Médica Nacional (BMN), como se le conoce, ha permitido a los bibliotecarios de dicha institución explorar el panorama actual de conocimientos y prácticas, y además los niveles de capacitación de colegas y usuarios de algunas bibliotecas que integran la Red de Bibliotecas Médicas cubanas, en su mayoría definidas con el término biblioteca híbrida, de gran resonancia en los últimos tiempos, ya que comprende aquellos servicios de información que se nutren de diversas herramientas y elementos tradicionales -mayormente representados por los soportes de información impresa y los catálogos en fichas-e incorporan las nuevas tecnologías para cumplir con sus objetivos -computadoras, redes y sistemas integrados de información, nuevos formatos, publicaciones electrónicas, etcétera-.La socialización entre bibliotecarios y usuarios ha permitido identificar roles y beneficios que ejecutan desde sus escenarios laborales. Los temas abordados en las conferencias se ajustan a problemáticas o conceptos tanto de la bibliotecología nacional como de la internacional.

Las conferencias que se imparten en la BMN tienen como objetivo fundamental que se conozcan los fundamentos científico-técnicos de una rama del saber humano, ya que estas conferencias desempeñan una función actualizadora, orientadora, metodológica y educativa, y se pueden desarrollar por cualquiera de los métodos de enseñanza existentes (Alfonso Sánchez, s.f.).

Desde la creación en 2013 de los espacios de conferencias, estos se han caracterizado por la unilateralidad de la comunicación, ya que por un lado hay un sujeto que habla y por otro hay varios sujetos que escuchan. Esto ha mantenido, hasta la actualidad, el propósito de aumentar los conocimientos o la comprensión del auditorio hacia un área determinada del saber y la autopreparacion del especialista conferencista, puesto quela tarea de desarrollar un tema determinado provoca la búsqueda de información retrospectiva y actual, sintetizar y utilizar técnicas escritas y orales que mantengan al público atento y motivado para tomar notas, intervenir mediante preguntas y debatir.

Para las instituciones de información, en este caso la BMN, la capacitación y entrenamiento permanente tiene gran importancia, y a todos los niveles constituye una de las mejores inversiones que puede hacerla organización, pues es una fuente de ventajas competitivas a corto y largo plazo y una de las principales fuentes de bienestar para el personal.

En cuanto a los aportes que va dejando en los profesionales de la BMN la capacitación en su modalidad de conferencia, se percibe como una ayuda para la toma de decisiones y solución de problemas, y que alimenta la confianza, la posición asertiva y el desarrollo, sube el nivel de satisfacción con el puesto trabajo, permite el logro de metas individuales, desarrolla un sentido de progreso en muchos campos, elimina los temores a la incompetencia o la ignorancia individual.

Teniendo en cuenta los objetivos de las conferencias semanales en la BMN como método de aprendizaje, se manifiestan algunos beneficios, y otros serán percibidos a largo plazo, como: las actitudes más positivas, el empoderamiento y conocimiento a todos los niveles, 
la promoción de la comunicación a nivel de toda la biblioteca, la reducción de la tensión y el manejo de áreas de conflictos, la agilización de la toma de decisiones y la solución de problemas, además del desarrollo de las personas mediante la promoción y la contribución a la formación de líderes y directivos.

El aprendizaje permanente puede comenzar en un aula presencial, semipresencial, llegar a ser virtual o en línea, pero lo cierto es que, una vez graduados, se logra un ambiente de autoestudio de mayor alcance que utiliza los conocimientos tecnológicos, comunicacionales e informacionales, que demuestran y generan un proceso de cambios en torno al aprendizaje de las personas. El aumento de los conocimientos que se consideran necesarios para realizar diversas actividades ha producido la necesidad de una demostración permanente de lo aprendido. Así, la experiencia es la base para el aprendizaje, y la reflexión es una parte importante del proceso de aprendizaje; la vía para expresarlo pueden ser las conferencias (Silvera Iturrioz, 2005).

Alfabetizar a las personas en las tecnologías de información y comunicación, así como en el manejo intelectual y crítico de la información obtenida gracias a dichas tecnologías, constituye una necesidad fundamental.

Las conferencias en su escenario proponen el vínculo entre bibliotecarios y usuarios, valorando esta vía para mostrar el rol que juega la biblioteca y el servicio de información que se ofrecen a profesionales de la salud que desconocían su valor y que, por lo tanto, ponían en peligro su posición dentro de la organización. La falta de sensibilización puede ser combatida con el alcance y la publicidad, y, de hecho, una serie de los participantes ya estaban participando en estas actividades (Kelham, 2014).

En su artículo "Professional development through attending conferences: reflections of a health librarían" ("Desarrollo profesional a través de asistencia a conferencias: reflexiones de un bibliotecario de la salud"), la escritora Ruth Jenkins (2015) de Berkshire Heathcare Foundation Trust, reflexiona sobre dos conferencias a las que asistió en 2014, LILAC y SLA. Jenkins, a través del proceso de reflexión, considera que los beneficios que aportan las conferencias pueden tributar una panorámica de conocimientos para los profesionales de la biblioteca y la información en el sector de la salud. En particular, discute las oportunidades y áreas de aprendizaje y desarrollo profesional que las conferencias pueden ofrecer, incluyendo la práctica basada en la evidencia y la conciencia actual, obteniendo nuevos conocimientos, objetividad, la creación de redes y los beneficios inesperados de las conferencias (Jenkins, 2015).

Para dar los primeros pasos, especialistas de la BMN tuvieron que responder a esta pregunta:

¿Cómo se realiza una conferencia?

Con experiencia en el terreno es válido puntualizar que para la preparación de una conferencia se deben tomar en consideración los siguientes pasos: Preparación de la conferencia, considerando aspectos tales como:

- Tema y tiempo.

- Justificación y Auditorio. Se selecciona a la persona capacitada para exponer un 
tema ante el auditorio. Esta persona puede o no pertenecer a la institución sede.

- La exposición del tema debe ser clara y completa.

- El auditorio permanece atento a la exposición.

El desarrollo de la conferencia incluye:

- Una introducción.

- La exposición de la teoría, apoyada con ejemplos, demostraciones o ilustraciones.

- Un periodo de preguntas.

- Finalmente, la síntesis del tema propuesto.

Elementos de una conferencia.

- El conferencista: normalmente es representante de una colectividad o de parte de un sector de una determinada colectividad, la que se convierte en su receptor colectivo y con la cual establece una relación jerárquica que lo convierte en un líder identificador de necesidades de información de su equipo.

- Tema o materia: la conferencia aborda asuntos de importancia o relevancia grupal, colectiva, o comunitaria.

- Objetivo: como finalidad, la conferencia busca comunicar una determinada concepción, visión o interpretación de temas y problemas que conciernen e importan a la vida de una colectividad para influir en ella, haciéndola tomar conciencia o reflexionar sobre los temas, orientándola o moviéndola a compartir visiones y metas, a tomar decisiones, acuerdos y/o a adoptar determinadas actitudes o comportamientos.

- Contexto y circunstancias de exposición: el discursante rellena prácticamente todos los espacios con palabras. Rara vez hace pausas de reflexión. El nivel formal del habla, los recursos verbales y no verbales, se utilizan para tener alcance y eficacia colectivos.

\section{Proceso para definir los temas y objetivos de las Conferencias en la BMN}

- Los objetivos y temas de las conferencias se formularán en función de las necesidades detectadas; estas pueden estar relacionadas con la calidad y desarrollar competencias informacionales, como por ejemplo: mejorar el clima organizacional, incrementar la productividad, aprovechar y agilizar procesos, aminorar los costos, elevar la calidad de los productos, mejorar el uso de los insumos, mejorar el desempeño, mejorar los procesos de trabajo, mejorar la atención al usuario, disminuir o reducir accidentes por fenómenos naturales, etc.

- Una vez definidos los temas, se identifican a los especialistas conferencistas según temáticas afines con su área, funciones, puestos de trabajo y tendencias actuales que definan cumplimientos de objetivos trazados por el área en cuestión.

- Se elabora un calendario anual que se visualiza en su espacio dentro de la página web de la BMN (http://www.bmns.sld.cu/conferencias-2017) 
- Se comprueba la infraestructura, que incluye escenarios como: salas de conferencia o de reuniones, sala de proyecciones, aulas de ejercicios y práctica, oficinas o ambientes administrativos. Se comprueba la disposición de requisitos de materiales, como ser: equipos de computación, proyectores, videos, equipos de sonido, televisores, retroproyectores, pizarras, láminas, plumones, etc.

- Se realiza el envío al correo electrónico de los usuarios por la lista de distribución de los temas del mes en curso. De esta forma se divulga y asegura la participación de los que deseen, según interés por el tema anunciado.

- Un aspecto que garantiza la organización y cumplimento de la actividad docente consiste en definir la logística que garantice la calidad. Para ello es necesario definir quiénes serán los responsables de la planificación, organización, conducción y evaluación de la capacitación y entrenamiento que se percibe en el público que asiste. Asimismo, es necesario definir y contar con la aprobación del conferencista anticipadamente, quien debe ser una persona idónea, con experiencia y conocimientos suficientes que garanticen una buena conferencia. Teniendo en cuenta esto, los conferencistas deben tener las siguientes características: conocimiento del tema, flexibilidad, adaptabilidad, sinceridad, sentido del humor, interés por el auditorio, didáctica clara y apropiada, puntualidad y entusiasmo; esto, sin dejar de puntualizar sobre la disposición y la motivación.

- El paso final, luego de concluida la conferencia, consiste en la entrega de la presentación utilizada a la Webmaster para que sea ubicada en el espacio Promoción Científica de la página web BMN. Así estará a disposición de todos los usuarios, asistentes y no asistentes.

A continuación, se detallan los procesos que llevan a cabo los especialistas conferencistas de la BMN, antes de presentar el tema al público que asiste cada martes de 2 p. m. a4 p. m., casi siempre en el escenario de la Sala de usos múltiples de la BMN.

Antes de la exposición se tienen en cuenta los siguientes puntos:

ConcientizaciónDesarrollo e investigaciónPreparación del Material (ppt, Word, video, Prezi, entre otros) Preparación de las instalaciones físicas Preparación psicológica Audiencia Presentación Personal Puntualidad Programación de la duración de la Conferencia.

Concientización: en este punto se debe ser consciente de la capacidad profesional para abordar el tema, es decir, si se tienen la experiencia y los conocimientos necesarios para dar la conferencia.

Desarrollo e investigación: es necesario siempre revisar nuevas fuentes de información para actualizarse, como así también buscar ejemplos sencillos y entendibles sobre el tema de la conferencia. Un mal concepto puede crear confusión y entorpece la exposición.

Preparación del material: como primer paso hay que hacer una indización del tema de la conferencia para mostrar las palabras claves o descriptores y así poder situar en un plano general el material de apoyo para la exposición.

Preparación de la síntesis en formato de presentación: luego de compilar y sintetizar la información recuperada con los descriptores o palabras claves, que puede definirse como 
la etapa más complicada, se procede a vincular: poder de síntesis, diseño, dominio de Microsoft Office para cada caso: Power Point, Word, PDF, MindManager o cualquiera de los productos de MultiMedia, Prezi, u otros.

Es sabido que las personas en una exposición totalmente verbal, sin apoyo audiovisual, mantienen una atención de un $60 \%$ inferior a una con apoyo audiovisual.

Preparación del material para los usuarios: antes de comenzar la exposición es conveniente entregar una copia del recurso informativo que se expondrá en pantallas o transparencias para que las personas que deseen tomar notas lo hagan, estas copias deben de tener al menos la mitad de la página con espacio para que los asistentes escriban sus notas; para esto es necesario aportar lápices o lapiceros junto con el material.

Preparación de las instalaciones físicas: las instalaciones físicas juegan un papel muy importante para conseguir una exposición exitosa, por lo que debe tenerse en cuenta eliminar todos los elementos de distracción posibles. El conferencista y la presentación deben ser fácilmente observables desde cualquier lugar de la audiencia. La audiencia debe estar ubicada con asientos $y$, de ser posible, con mesas. La temperatura debe ser adecuada: ni caliente, que provoque sofoco, o que se duerma la audiencia, ni tampoco se debe llegar al extremo de que la gente esté pasando mucho frío, lo que genera a su vez mucha distracción. La iluminación debe ser adecuada tanto en el área de exposición como en la audiencia, pues es importante que el conferencista tenga contacto visual con la audiencia y que ésta tenga la iluminación suficiente para poder escribir. El equipo de sonido es muy importante en audiencias grandes, al igual que el equipo de computación, pantallas, proyectores y otros audiovisuales requeridos. Una vez que la conferencia comienza no es conveniente que nadie pase por el lugar instalando, cargando, repartiendo o cualquier otra actividad que distraiga al auditorio. Al igual que evitar cualquier otro elemento de distracción como celulares, música ambiental, y otros ruidos. Es muy importante la idea de tener un reloj de espalda a la audiencia y de frente al expositor para tomar el tiempo real de la conferencia, que no debe exceder los 45 minutos, y que a su vez le ayude a dirigirlo.

Preparación psicológica: es necesario que el conferencista esté consciente de su capacidad profesional para abordar el tema de su conferencia y de poder responder a las preguntas que le puedan hacer. Para ello debe asegurarse de que sus conocimientos sean suficientes o de contar con alguno de sus compañeros que lo asista cuando así lo crea necesario. Para eso el equipo de exposición debe ponerse de acuerdo para que cuando exista alguna situación, ya sea por preguntas o cualquier motivo, el expositor haga alguna seña específica para solicitar ayuda, de lo contrario no es correcto que nadie intervenga pues le quita postura y le resta credibilidad frente a la audiencia. Tomarse unos minutos para lograr una adecuada concentración.

Audiencia: es muy necesario saber el nivel profesional, actividad laboral y a qué se dedica la audiencia. También, si es posible, tener acceso a todos los nombres para familiarizarse con ellos; sería muy conveniente además relacionar las ubicaciones con los nombres de cada persona dentro del auditorio; esto con el fin de que en algún momento el expositor se pueda dirigir de forma más personalizada al público. Un factor muy importante es saber 
qué espera la audiencia de nuestra conferencia para unirlos con nuestros objetivos; se inicia por tanto con la identificación de cada especialista, el área a la cual pertenece y funciones que realiza. Al finalizar se debate el tema y se reflexiona teniendo en cuenta escenarios o contextos de actuación.

Presentación personal: es muy importante la presentación personal del conferencista. Hay que intentar ubicarse a la misma altura de la audiencia y tomar en cuenta el lugar desde donde se va a exponer.

Puntualidad: es un requisito ejecutivo, si una gran parte de la audiencia ha llegado temprano, por respeto a ellos la conferencia debe comenzar a la hora programada (las 2 p. m.), no esperando a los que llegan tarde (sólo en el caso de que se espere a una persona muy importante).

Programación de la duración de la exposición: es sabido que una idea corta no debe de durar más de 15 minutos de exposición, en el caso de una idea mayor el tiempo más recomendable es de 45 minutos, después de este tiempo hay mucha probabilidad de pérdida de atención. Solo en el caso de una audiencia ya previamente avisada los tiempos pueden ser mayores, pero hay que tener en cuenta que es conveniente dar descansos cada 2 horas con algún refresco, té o café para reanimar un poco a los concurrentes.

El aporte de información sintetizada para describir escenarios actuales de la actividad bibliotecaria, está recogida a continuación en una selección realizada del listado de conferencias impartidas por especialistas de la bibliotecología y ciencias de la información de la BMN y otras bibliotecas de la Red, así como usuarios profesionales de la salud, desde 2013 hasta 2016.

\section{Diciembre 2016}

"¿Qué es y qué implicaciones tiene altmetrics?" Ponente: Lic. Sonia Santana Arroyo/Especialista Principal DSI

"La propiedad intelectual y los permisos de reproducción y derechos de imagen." Ponente: Lic. Lisbeth Cruz García/] 'Grupo Conservación

"La importancia de desarrollar tu marca personal como bibliotecario." Ponente: Dra.C. Maria del Carmen González Rivero/J' Grupo Servicios Bibliotecarios

\section{Noviembre 2016}

"¿Qué características poseen los usuarios de la Web 2.0?" Ponente: Lic. Yamila Ricardo Sánchez/Especialista del Servicio de Bibliografía

"El catálogo de la biblioteca: ¿esencia u obsolencia?" Ponente: Lic. YanetLujardo Escobar/Jefa de Grupo Procesos Técnicos

"Nuevos desafíos para el profesional de la información en el entorno ALFIN." Ponente: Téc. Cristina González Pagés/Asesora Técnica y Editora Web

\section{Octubre 2016}

"Soy Bibliotecario y no encuentro nada..." Ponente: Lic. Tania M. González Díaz/Especialista Sala de Navegación 
"Herramientas Eficientes para Optimización de Soportes Fijos y Portables." Ponente: Dr.C Ing. Luis F de la Cruz Figueroa/ICBP "Victoria de Girón"

\section{Septiembre 2016}

"Perfiles profesionales 2.0: más allá del contentcurator y el bibliotecario community manager." Ponente: Lic. Adriana M. Perera González/Especialista Servicio de Bibliografía

"BIBLIO-INFORMÁTICOS. Nuevos roles en la era digital." Ponente: Téc. Cristina González Pagés/Asesora Técnica y Editora Web

\section{Julio 2016}

"Del IndexMedicus a la actualidad." Ponente: Lic. Ana Luisa Pinillo León/Especialista del Servicio de DSI

"Los Knowmads en la sociedad." Ponente: Dra.C Maria del Carmen González Rivero/Jefa de Servicios Bibliotecarios

\section{Junio 2016}

"Programa internacional de Advocacy de la IFLA." Ponente: Lic. Bárbara Lazo Rodríguez/Miembro Comité Permanente IFLA LAC

"El bibliotecario frente al saber: aportes del pensamiento de Newman." Ponente: MSc. Ileana Armenteros Vera/Jefa Dpto. Servicios Bibliotecarios

\section{Mayo 2016}

"¿Cómo construyo el DOI de mi biblioteca?" Ponente: Téc. Mónica Talavera Castro/Especialista del Área de Digitalización

"En una Biblioteca, ¿Cómo deben vestir los usuarios y bibliotecarios?" Ponente: Téc. Alina Brito Nodal/Recepcionista de la BMN

\section{Abril 2016}

"Ventajas de la Seguridad Informática en las Bibliotecas." Ponente: Téc. Cristina González Pagés/Asesora Técnica y Editora Web

"¿Por qué no tengo usuarios en la Biblioteca?" Ponente: Lic. Sonia Santana Arroyo/Especialista Principal del Servicio DSI

\section{Marzo 2016}

"¿Necesito servicios de calidad en mi Biblioteca? ¿Por dónde empiezo?" Ponente: Dra.C. Maria del Carmen González Rivero/J 'Grupo Servicios al Público

"¿Qué busco cuando hago control de calidad a los catálogos de mi biblioteca?" Ponente: Lic. Yanet Lujardo Escobar, Téc. Elizabeth Ramírez Herrera/Especialistas de Procesos Técnicos

\section{Enero 2016}

"Terremoto: enemigo letal." Ponente: Lic. Lisbeth Cruz García/Departamento de Conservación 


\section{Año 2015}

\section{Noviembre 2015}

"El '5' por una buena bibliografía." Ponente: Lic. Adriana Ma. Perera González/Especialista del Servicio Bibliografía

"Resiliencia: construyendo en adversidad." Ponente: Dra.C. Maria del Carmen González Rivero/Especialista de Servicios Bibliotecarios

\section{Octubre 2015}

"Desastres en Bibliotecas." Ponente: Téc. Roberto Carballo Vega/Departamento de Conservación

\section{Julio 2015}

"Mendeley: ventajas y desventajas para la bibliografía." Ponente: Lic. Adriana Ma. Perera González/Especialista del Servicio Bibliografía

"La paradoja de la sociedad de la información: la desinformación." Ponente:Lic. Yamila Ricardo Sánchez/Especialista del Servicio Sala de Lectura

\section{Junio 2015}

"¿Por qué hay usuarios que naufragan?" Ponente: Lic. Tania González Díaz/Especialista del Servicio Sala de Navegación

"¿Qué tenemos identificado que necesita un usuario potencial?" Ponente: Lic. Sonia Santana Arroyo/Especialista del Servicio DSI

\section{Mayo 2015}

"El libro electrónico en Sala de Lectura. Qué prefieren los usuarios? ¿Impreso o digital?" Ponente: Lic. Yamila Ricardo Sánchez/Especialistas del Servicio Sala de Lectura

"Una bióloga desarrolla habilidades para ofrecer el servicio de Bibliografía en la BMN." Ponente: Lic. Adriana Perera González/Especialista del Servicio Bibliografía

\section{Abril 2015}

"El biodeterioro de documentos. Alternativas para el control de plagas." Ponente: Lic. Lisbeth Cruz García/Especialista de Procesos Técnicos.

"Notimed: Noticias de Salud acerca de Cuba." Ponente: Lic. Ana Luisa Pinillo/Especialista del Servicio DSI.

\section{Marzo 2015}

"PREZI: para las futuras presentaciones y mapas conceptuales." Ponente: Téc. Cristina González Pagés/Editora web y asesora técnica de Servicios Bibliotecarios.

"¿Cómo influye el bibliotecario en las búsquedas en Internet de los usuarios?" Ponente: Lic. Tania González Díaz/Especialista del Servicio Sala de navegación.

"Productos Informativos para la toma de decisiones del Directivo de Salud." Ponente: Lic. Sonia Santana Arroyo/Especialista del Servicio DSI. 


\section{Febrero 2015}

"Cómo, qué y cuánto aportan los usuarios al procedimiento de Sala de Lectura." Ponente: Lic. Yamila Ricardo Sánchez/Especialista del Servicio de Sala de Lectura.

\section{Noviembre 2014}

"La Entrevista: método de interrelación usuario-profesional de la información." Ponente:

Lic. Tania M. González Díaz/Especialista del Servicio Sala de Navegación.

"¿Sabe usted cómo citar sitios web?" Ponente: Lic. Sonia Santana Arroyo/Especialista del Servicio DSI.

\section{Octubre 2014}

"Vocabulario controlado en la recuperación de información." Ponente: Lic. Ana Luisa Pinillo León/Especialista del Servicio DSI.

"Rediseño de un servicio comunitario: BiblioSIDA." Ponente: Lic. Raisa Alayo Morales/Especialista del Servicio de Bibliografía.

\section{Septiembre 2014}

"ILN: Un proyecto internacional para el intercambio entre bibliotecarios." Ponente: Lic. Adriana M. Perera González/Especialista del Servicio DSI.

"Herramientas sociales para investigadores." Ponente: Lic. Sonia Santana Arroyo/Especialista del Servicio DSI.

\section{Agosto 2014}

"Presentación de la Base de Datos SPRINGER LINK." Ponente: Lic. Tania M. González Díaz/Especialista del Servicio de Referencia.

"¿Se elimina el acceso a Google? ¿SI o NO?." Ponente: Lic. Margarita Pobea Reyes/Especialista del Servicio de Sala de Navegación.

\section{Julio 2014}

"La comunicación con los usuarios. Nuevos retos" Ponente: Lic. Ana Liz García Meriño/Especialista del Servicio de Referencia.

\section{Junio 2014}

"Fuentes de Información. Literatura Gris" Ponente: Lic. Tania González Díaz/Especialista del Servicio de Referencia

\section{Mayo 2014}

"ClinicalKey" Ponente: Lic. Ana Liz García Meriño/Especialista del Servicio de Referencia

\section{Abril 2014}

"Redes sociales académicas" Ponente: Lic. Sonia Santana Arroyo/Especialista del Servicio $\underline{\mathrm{DSI}}$ 
"Publicaciones científicas" Ponente: Lic. Tania González Díaz/Especialista del Servicio de Referencia

\section{Marzo 2014}

"Ética en bibliotecas" Ponente: Lic. Ana Luisa Pinillo León/Especialista del Servicio DSI

"SeCiMed: Catálogo Colectivo de Publicaciones Seriadas en Ciencias de la Salud" Ponente: Lic. Ana Liz García Meriño/Especialista del Servicio de Referencia

\section{Febrero 2014}

"¿Cómo impresionar al usuario?" Ponente: Lic. Margarita Pobea Reyes/Especialista del Servicio DSI

"Calidad y bibliotecas" Ponente: Lic. Tania M. González Díaz/Especialista del Servicio de Referencia

\section{Enero 2014}

"Información Factográfica: Datos y gráficos para hablar de salud." Ponente: Lic. Adriana Perera González/Especialista del Dpto. DSI

"Trabajo en equipo" Ponente: Lic. Ana Liz García Meriño/Especialista del Servicio de Referencia

\section{Año 2013}

"Etapas de un proceso de investigación" Ponente: Lic. Adriana Perera González / Especialista del Dpto. de DSI

"El artículo científico. Proceso de redacción y publicación" Ponente: Lic. Sonia Santana Arroyo / Especialista del Dpto. de DSI

"Marketing en bibliotecas médicas" Ponente: Lic. Tania M. González Díaz / Especialista del Servicio de Referencia

"Herramientas útiles para el trabajador del conocimiento." Ponente: Lic. Ana Liz García Meriño / Especialista del Servicio de Referencia

\section{Las Conferencias en cifras}

A continuación, se realiza un análisis de información graficada que tiene en cuenta cifras de conferencias y asistencia por años, desde el 2013 al 2016.

El Gráfico 1 muestra los inicios de la actividad capacitadora con el diseño de una conferencia mensual en el 2013, para un total de 12, sin contabilizar la asistencia porque no se contaba con el registro de asistencia. 


\section{Gráfico 1. Total de conferencias del año 2013-2016}

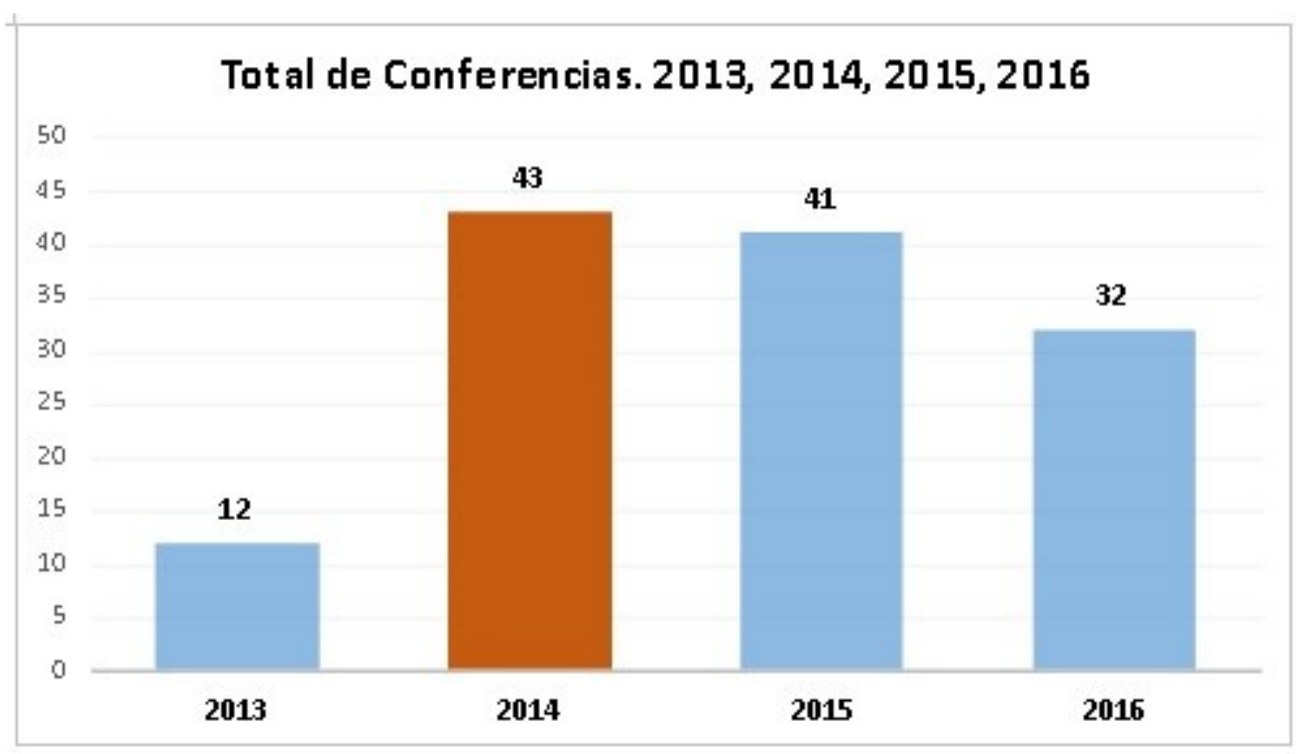

Para el año 2014 (Gráfico 2) se planificaron las conferencias mensuales cada martes. Con el registro de asistencia se hizo posible contabilizar y evaluar el alcance e interés de los temas propuestos.

Para un total de 43 conferencias se registra un total de 619 asistentes, siendo mayo y julio los de mayor asistencia.

\section{Grafico 2. Estadística de conferencias y asistencia en el año 2014}

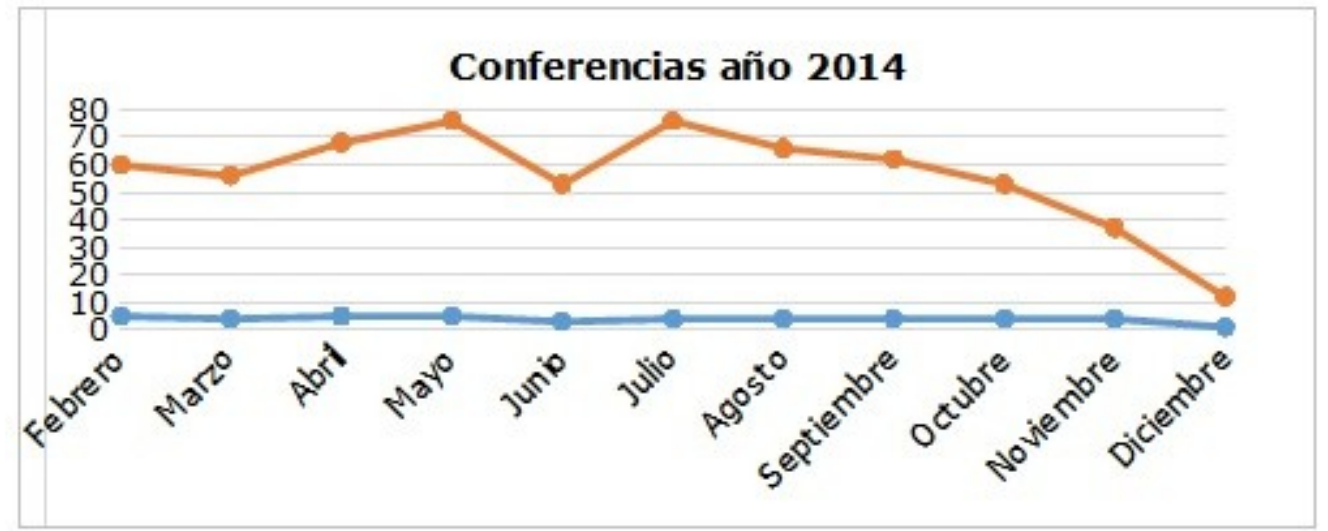

Para el año 2015 (Gráfico 3) se planificaron las conferencias mensuales, y para un total de 41 conferencias se registra un total de 593 asistentes. Siendo abril el de mayor asistencia registrada, con 110 bibliotecarios y usuarios, y con la menor asistencia los meses de febrero y agosto, este último justificado por ser la etapa vacacional en los centros educativos y personal que labora en bibliotecas. 


\section{Gráfico 3. Estadística de conferencias y asistencia en el año 2015}

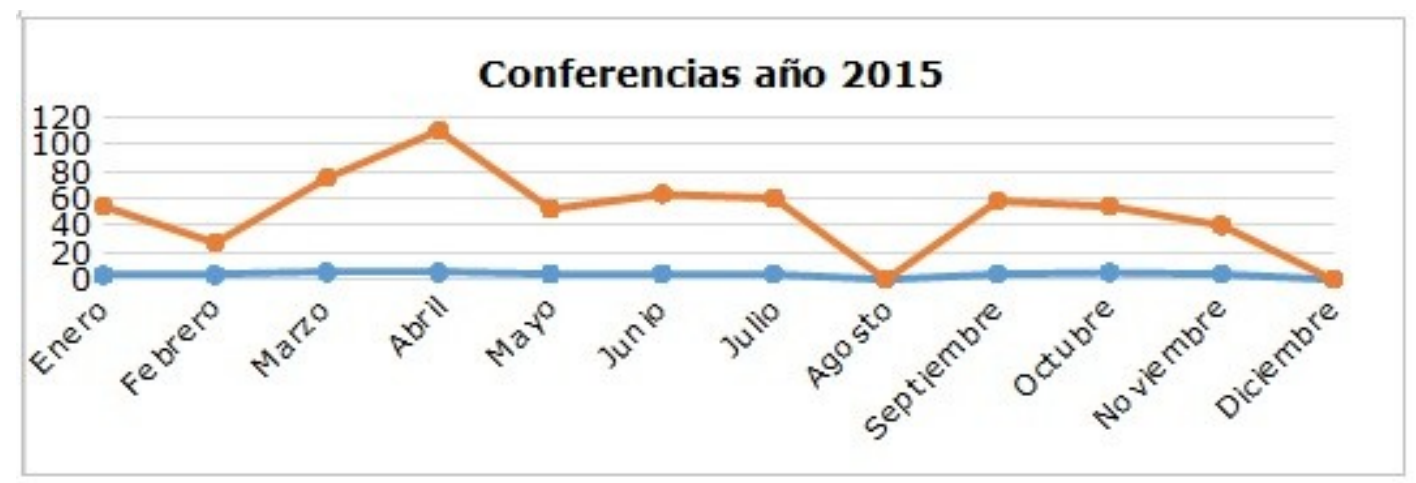

Para el año 2016 de enero a noviembre (Gráfico 4) las conferencias planificadas mensuales fueron 28, figurando en los registros de asistencia un total de 348 asistentes. Siendo mayo el de mayor asistencia registrada con 60 bibliotecarios y usuarios, para el mes de febrero y agosto se decidió no planificar conferencias, debido a un reordenamiento en la estructura organizacional de la BMN y la planificación de vacaciones de algunos especialistas.

\section{Gráfico 4. Estadística de conferencias y asistencia en el año 2016 (enero- noviembre)}

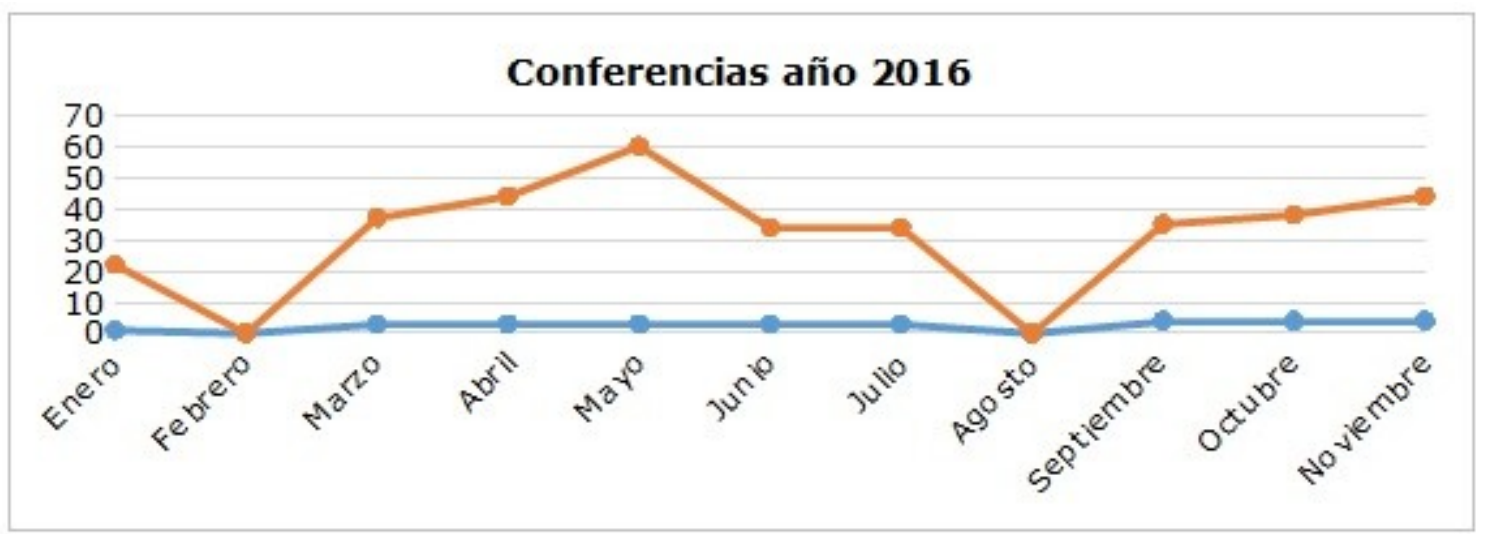

Siguiendo las cifras en años es preocupante el decrecimiento en la asistencia de los bibliotecarios: cuando para el 2014 se muestra una cifra de 619 asistentes, para el 2015 una asistencia anual de 593, cerrando el año 2016 con una asistencia de 348 profesionales que asistieron para el intercambio de conocimiento y comparar contextos nacionales e internacionales en el ámbito bibliotecario.

El decrecimiento ha sido tema de análisis por parte de especialistas que lideran la actividad. Destacan dos criterios relacionados: el primero relacionado con los temas seleccionados para las conferencias, que reflejan el ámbito internacional bibliotecario y con poca visión o aplicación en el contexto nacional; el segundo criterio tiene que ver con la interrogante: ¿cuánto representa en la evaluación y curriculum profesional asistir a las 
conferencias?

\section{Conclusiones}

Aunque los objetivos del espacio de aprendizaje con las conferencias están bien definidos, tomando en cuenta la necesidad real de desarrollo profesional, resulta cierto que aún no se considera una actividad de formación entre bibliotecarios de la red de bibliotecas médicas, porque no se ha logrado despertar el interés, sin corte de obligación, en especialistas de otras instituciones de información científica que presenten temas contextuales para las mayorías. La asistencia a las conferencias que se realizan en la BMN, cuenta, por lo general, con la participación de los especialistas de la propia institución que convoca, y los que asisten suelen tener poca intención de debatir el tema que se presenta, pues con la asistencia puede resultarles suficiente. En cuanto al debate, sugerencias para el cambio o nuevas ideas, suele ser mínimo, pues prevalecen criterios que en escasas ocasiones se logran llevar a la práctica. Hay que sumar a este evento, como punto negativo, la demoledora presencia del agente "desmotivación", que colma muchos espacios de actividades de intercambio de conocimientos que se realizan en aras de no perder el protagonismo que la Sociedad de la Información ha puesto en manos de los bibliotecarios. A partir del análisis de los aspectos más negativos que frenan la asistencia y participación en las conferencias como son: el horario, frecuencia, temas y espontaneidad, puede resultar positivo un rediseño del espacio Conferencias en la Biblioteca Médica Nacional (BMN), teniendo en cuenta elementos que motiven e incentiven al profesional de la información en el deseo de involucrarse activamente en el escenario bibliotecológico contemporáneo.

\section{Bibliografía}

Alfonso Sánchez, I. (s.f.). Elementos a considerar en la preparación metodológica de una Conferencia. Recuperado de http://www.sld.cu/galerias/pdf/sitios/hematologia/pasos_para_ejercicio docente.pdf

Hernández Pacheco, F., y Quezada Escamilla, D. (2016). Los recursos humanos en las bibliotecas públicas de México: una investigación exploratoria. Investigación $\begin{array}{lrl}\text { Bibliotecológica, } & \text { 30(68). Recuperado }\end{array}$

http://www.sciencedirect.com/science/article/pii/S0187358X16000034

Jenkins, R. (2015). Professional development through attending conferences: reflections of a health librarian. Health Information \& Libraries Journal,32(2),156-60. Recuperado de http://onlinelibrary.wiley.com/doi/10.1111/hir.12101/full

Kelham, C. (2014). Health care librarians and information literacy: an investigation. Health Information \& Libraries Journal, 31(3), 235-238. Recuperado de http://onlinelibrary.wiley.com/doi/10.1111/hir.12071/full

Petrinic, T., y Urquhart, C. (2007). The education and training needs of health librariansthe generalist versus specialist dilemma. Health Information \& Libraries Journal, 24(3), 167-176. Recuperado de http://onlinelibrary.wiley.com/doi/10.1111/j.14711842.2007.00717.x/full 
Silvera Iturrioz, C. (2005). Los bibliotecarios en la sociedad de la información. ACIMED, 13(3). Recuperado de http://bvs.sld.cu/revistas/aci/vol13 3 05/aci07305.htm

Spring, H., Kunkel, M., Gilman, I., Henderson, N., y White, J. (2016). International collaborations in learning and teaching: perspectives from a visiting professorship. Health Information \& Libraries Journal, 33(3), 249-254. Recuperado de http://onlinelibrary.wiley.com/doi/10.1111/hir.12148/full 